ARTICLE

\title{
Early DMO: a predictor of poor outcomes following cataract surgery in diabetic patients. The DICAT-II study
}

Giacomo Panozzo (iD ${ }^{1,2}{ }^{凶}$, Giulia Dalla Mura ${ }^{2}$, Elia Franzolin (iD ${ }^{3}$, Diana Giannarelli ${ }^{4}$, Valeria Albano ${ }^{5}$, Gianni Alessio ${ }^{5}$, Alessandro Arrigo ${ }^{6}$, Stefano Casati ${ }^{3}$, Paola Cassottana ${ }^{7}$, Cecilia Contardi ${ }^{8}$, Rossella D'Aloisio iD ${ }^{8}$, Francesco Fasce ${ }^{6}$, Elena Gusson ${ }^{3}$, Giorgio Marchini ${ }^{3}$, Leonardo Mastropasqua ${ }^{8}$, Massimo Niccolò ${ }^{9}$, Carmela Palmisano ${ }^{5}$, Marco Rocco Pastore (iD ${ }^{10}$, Sandro Saviano ${ }^{10}$, Daniele Tognetto ${ }^{10}$ and Francesco Bandello ${ }^{6}$

(c) The Author(s), under exclusive licence to The Royal College of Ophthalmologists 2021

BACKGROUND: The prospective Dlabetes and CATaract Study II (DICAT II) was performed to characterise the risks of cataract surgery to the retinae of patients with early diabetic macular oedema (E-DMO).

METHODS: DICAT II was a prospective, comparative, multicentre, observational study involving six Italian clinics. Patients were aged $\geq 55$ years, had type 1 or 2 diabetes with spectral-domain optical coherence tomography evidence of ESASO classification Early DMO. Group 1 eyes (78 eyes, 78 patients) underwent phacoemulsification-based cataract surgery. Group 2 eyes (65 eyes, 65 patients) had E-DMO and either clear media or had undergone uncomplicated cataract surgery $\geq 1$ year previously. Central subfield thickness (CST) and best-corrected visual acuity (BCVA) were assessed in both groups.

RESULTS: The negative impact of surgery on CST was evident after the first postoperative week; CST peaked during the first month, then rapidly decreased. CST worsening $\geq 10 \mu \mathrm{m}$ was observed in 63/78 eyes (80.7\%) and 29/65 eyes (44.6\%) in Groups 1 and 2 , respectively $(p<0.0001)$. CST worsening of $\geq 50 \mu \mathrm{m}$ was observed in 51 eyes $(65.4 \%)$ and 10 eyes $(15.4 \%)$ in Groups 1 and 2 , respectively $(p<0.0001)$. Mean CST worsening was lower in Group 2 than in Group $1(38.6 \pm 30.4 \mu \mathrm{m}$ vs $85.5 \pm 55.3 \mu \mathrm{m}, p<0.0001)$ with a lower BCVA loss $(-2.6 \pm 3.5$ letters vs $-8.2 \pm 6.2$ letters, $p<0.0001)$. Higher glycaemic levels and HBA1c levels were significantly associated with the risk of $>50 \mu \mathrm{m}$ CST worsening in eyes from both groups.

CONCLUSION: Early DMO is associated with poorer outcomes after cataract surgery and requires close pre- and postoperative monitoring.

Eye (2022) 36:1687-1693; https://doi.org/10.1038/s41433-021-01718-4

\section{INTRODUCTION}

The prevalence of diabetes is high and is projected to continueincreasing. In 2019, the International Diabetes Federation estimated that 240 million people were living with the disease (a prevalence of $9.6 \%$ ): this will rise to 356 million (11.1\% prevalence) in 2045 [1]. Moreover, this prevalence strongly increases with age, rising to over $20 \%$ in elderly subjects $[2,3]$. Diabetes is pernicious: chronic hyperglycaemia affects cardiovascular, renal and neural systems. The visual system is no exception, where the disease is associated with damage of many ocular tissues, particularly the retina, but also the crystalline lens: a large proportion of people with diabetes will experience accelerated cataract development and require cataract surgery more frequently and at an earlier age compared with non-diabetics [4].

Diabetic macular oedema (DMO) is one frequent complication in people with diabetes: around $11 \%$ of all people with diabetes in Europe develop it [5]. Hyperglycaemia damages the retinal vasculature and degrades the blood-retinal barrier, leading to fluid leaking from the vessels into the neurosensory retina, resulting in oedema and vision loss [6]. A recent large prospective clinical study performed by our group (the Dlabetes and CATaract Study - DICAT I) demonstrated that $20.4 \%$ of all subjects undergoing cataract surgery are diabetic [7].

Cataract surgery in people with diabetes can be associated with the development or worsening of macular oedema, secondary to an increase in inflammatory mediators in the aqueous and vitreous after phacoemulsification. The risk of worsening of preoperative DMO after surgery, thereby reducing or even eliminating the visual benefits of cataract extraction is well known, and has prompted the use of prophylactic therapeutic measures $[8,9]$. The risk of post-cataract surgery macular oedema development in diabetic eyes without preoperative maculopathy was unclear until a British retrospective database study [10] that employed an electronic medical record dataset containing over

\footnotetext{
${ }^{1}$ Ophthalmology Unit, Clinica San Francesco, Verona, Italy. ${ }^{2}$ ESASO, European School of Advanced Studies in Ophthalmology, Lugano, Switzerland. ${ }^{3}$ Ophthalmology Unit, Department of Neurosciences, Biomedicine and Movement, University of Verona, Verona, Italy. ${ }^{4}$ UOSD Clinical Trial Center, Biostatistics and Bioinformatics, Regina Elena National Cancer Institute IRCCS, Rome, Italy. ${ }^{5}$ Department of Medical Science, Neuroscience and Sense Organs, Eye Clinic, University of Bari, Bari, Italy. ${ }^{6}$ Department of Ophthalmology, IRCCS San Raffaele Scientific Institute, Milan, Italy. ${ }^{7}$ Department of Ophthalmology, San Martino University Hospital, IRCCS, Genova, Italy. ${ }^{8}$ Department of Ophthalmology, G. d'Annunzio University of Chieti-Pescara, Chieti, Italy. ${ }^{9}$ Department of Ophthalmology, University of Genova, Genova, Italy. ${ }^{10}$ Department of Medical Surgical Sciences and Health, Eye Clinic, University of Trieste, Trieste, Italy. ${ }^{\circledR}$ email: g.panozzo@iol.it
} 
80,000 subjects finally demonstrated that such eyes have a significantly increased relative risk of developing macular oedema after cataract surgery compared with normal eyes, and that this risk increases with the level of retinopathy: the relative risk was $1.8 \%$ in eyes without any form of retinopathy, but this rose to $6.6 \%$ in eyes with advanced retinopathy [10].

The impact of cataract surgery on the retinae of diabetic eyes has been characterised in both eyes with full-blown DMO and those with normal maculae, but no reports exist of its impact on eyes that display only the initial signs of DMO. This form of minimal oedema, usually called 'pre-clinical' or 'non-significant' DMO, was recently termed 'Early DMO' (E-DMO) in a recent optical coherence tomography (OCT)-based morphologic classification by an international panel of experts (the European School for Advanced Studies in Ophthalmology [ESASO] DME Classification; see Methods for definition) [11]. In DICAT I, E-DMO was by far the most frequent type of DMO observed in diabetic patients undergoing cataract surgery, being present in $20.9 \%$ of enrolled patients [7].

To better characterise how cataract surgery and the associated increase in inflammatory mediators affects the retina in eyes with preoperative E-DMO, we performed the prospective Dlabetes and CATaract Study II (DICAT II).

\section{METHODS}

DICAT II was a prospective, comparative, multicentre, observational study that initially involved 19 Italian clinical sites, but the COVID-19 pandemic interrupted the follow-up of most study participants in 12 of these sites, which were excluded from the data analysis. Six centres began recruitment before the start of the pandemic and were able to complete the study with a very limited loss to follow-up, producing the statistically relevant data reported here.

The study protocol was approved by the Ethical Committee of each participating site and was aligned with the tenets of the Declaration of Helsinki. Each participant gave written informed consent to participate in the study. The DICAT II Study is listed on Clinicaltrials.gov with accession number NCT04096131.

\section{Study population}

Eligible study participants aged $\geq 55$ years and had type 1 or type 2 diabetes with spectral-domain (SD)-OCT evidence of E-DMO according to the ESASO morphologic classification (see Study design for definition). Any level of diabetic retinopathy, even if laser-treated $\geq 1$ year before, was admitted and recorded according to ESASO classification [11].

\section{Study groups}

Group 1 (treatment group) consisted of eyes with E-DMO scheduled for cataract surgery. Group 2 (control group) comprised eyes with E-DMO and clear media and/or pseudophakia (non-complicated cataract surgery performed at least 1 year before), or cataract surgery scheduled after the end of follow-up. Some study participants with bilateral E-DMO were able to participate in both groups: one eye was scheduled for cataract surgery (Group 1); the other eye served as control (Group 2).

\section{Study design}

Participants in Group 1 were followed for 4 months before surgery (baseline, 2-4 months) and a further 4 months after surgery (1-2 weeks, 1-2-3-4 months) for a total of 8 months. Group 2 participants were followed for 8 months at 2-month intervals (baseline and 2-4-6-8 months). In patients with bilateral DMO, the evaluation of both eyes followed the schedule of Group 1. Patients in this analysis were enrolled from November 2019 to April 2020.

\section{Definition of Early DMO}

E-DMO was defined as follows: small intraretinal cysts associated with wellrecognisable and normal retinal segmentation (inner retinal layers, ellipsoid zone, external limiting membrane), with increases in central subfield thickness (CST) $<30 \%$ of maximum normal values. The intraretinal cysts are defined as round, minimally reflective spaces within the neurosensory retina, located in the outer nuclear layer, inner nuclear layer or ganglion cell layer. Cyst size was graded considering the dimension of the largest intraretinal cyst identifiable on the scan.

The ESASO Classification [11] standard figure number 1 was used for case selection by each participating centre (Fig. 1). The OCT images of each eye were sent to the coordinating centre and the presence of E-DMO was confirmed before that eye could be admitted to the study.

\section{Identification of eligible subjects}

All patients eligible for Group 1 and a proportion of patients included in Group 2 were identified during regular preoperative cataract examinations, with the remainder of Group 2 patients being enrolled during routine OCT screening of diabetic patients. All patients underwent an OCT scan according to the protocol listed below.

\section{Inclusion criteria}

1. Patients with cataract and E-DMO in just one eye (Group 1).

2. Patients with bilateral cataract and bilateral E-DMO. Both eyes were enrolled: one eye received surgery after 4 months of follow-up (Group 1), the other after the end of follow-up (Group 2).

3. Patients with bilateral E-DMO and cataract in just one eye. Both eyes were enrolled. If the eye with E-DMO and no cataract was pseudophakic, it was eligible in Group 2 only if surgery was performed at least 12 months prior to enrolment.

4. Patients with cataract and no E-DMO in the eye scheduled for surgery but E-DMO in the other eye. Only the eye with E-DMO was enrolled in Group 2.

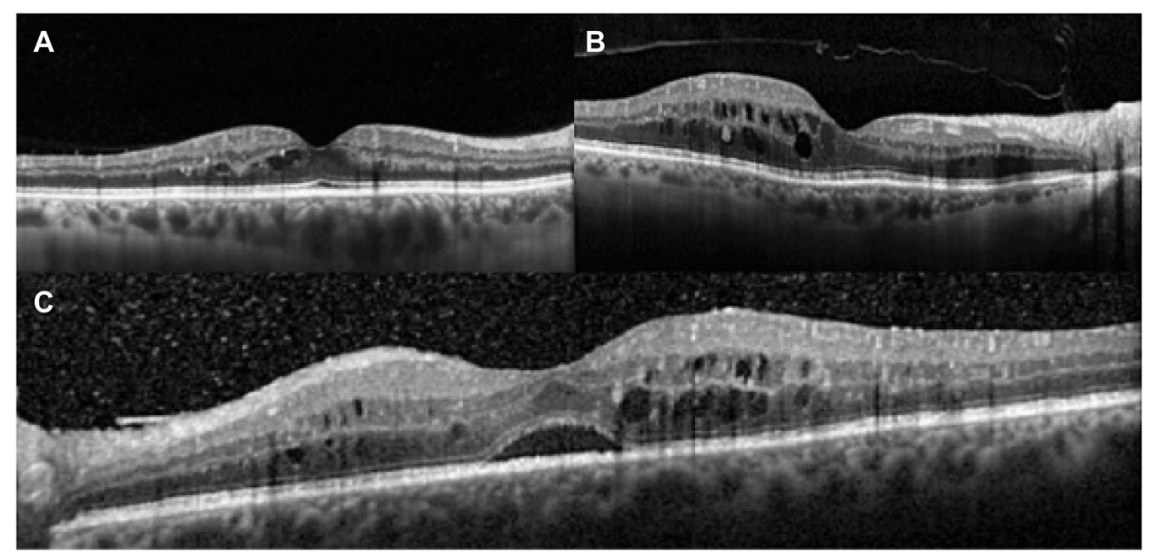

Fig. 1 The ESASO Classification standard. Early DMO (E-DMO) is defined as small intraretinal cysts associated with well-recognisable and normal retinal segmentation (inner retinal layers, ellipsoid zone and external limiting membrane) and with an increase in central subfield thickness (CST) less than $30 \%$ of maximum normal values. A-C represent three levels of progression still considered E-DMO. 
Table 1. Baseline characteristics of the two groups.

\begin{tabular}{|c|c|c|c|c|}
\hline & Total & Group 1 & Group 2 & Difference ( $p$ value) \\
\hline Eyes & 143 & 78 & 65 & - \\
\hline Age (years) & $71.2 \pm 6.9$ & $72.7 \pm 5.1$ & $68.7 \pm 8.1$ & 0.052 \\
\hline Gender, Male:Female (\%) & $60.1: 39.9$ & $60.3: 39.7$ & $60.0: 40.0$ & 0.9751 \\
\hline Duration of DM (years) & $14.3 \pm 9.3$ & $12.8 \pm 7.2$ & $16.1 \pm 11.0$ & 0.0330 \\
\hline Median (range) & $13(1-52)$ & $12(1-35)$ & $14(2-52)$ & \\
\hline Insulin intake (\%) & 37.5 & 33.3 & 40.8 & 0.0415 \\
\hline Glycemia (mg/dL) & $128.6 \pm 31.8$ & $129.3 \pm 31.3$ & $127.8 \pm 32.6$ & 0.7799 \\
\hline $\mathrm{Hb} 1 \mathrm{Ac}(\mathrm{mmol} / \mathrm{mol})$ & $52.8 \pm 10.1$ & $51.9 \pm 11.3$ & $53.9 \pm 8.5$ & 0.2415 \\
\hline \multicolumn{5}{|l|}{$D R$ grade (\%) } \\
\hline Absent & 17.5 & 32.1 & 28.4 & 0.041 \\
\hline Mild NP & 39.2 & 34.6 & 26.2 & 0.054 \\
\hline Moderate NP & 33.6 & 29.5 & 28.5 & 0.33 \\
\hline Advanced NP & 9.8 & 3.8 & 16.9 & 0.02 \\
\hline Proliferative & 0 & 0 & 0 & \\
\hline BCVA, ETDRS (letters) & $32.4 \pm 5.7$ & $32.4 \pm 5.7$ & $48.0 \pm 5.5$ & $<0.0001$ \\
\hline CST $(\mu \mathrm{m})$ & $293.2 \pm 38.4$ & $291.6 \pm 44.5$ & $295.1 \pm 29.8$ & 0.5894 \\
\hline
\end{tabular}

$B C V A$ best-corrected visual acuity, CST central subfield thickness, DM diabetes mellitus, DR diabetic retinopathy, ETDRS Early Treatment Diabetic Retinopathy Study, NP non-proliferative.

Bold entries show the difference of the other baseline parameters was considered not significant except for BCVA, where the difference between the two groups was highly significant.

\section{Exclusion criteria}

1. Past ocular surgery of any kind (except for cataract surgery performed at least 1 year before in Group 2) or ocular pathologies other than diabetic retinopathy.

2. Macular oedema considered by the investigator to be due to a cause other than diabetes.

3. OCT examination evidencing vitreoretinal interface abnormalities (e.g., taut posterior hyaloid or epiretinal membrane) being considered as the primary or a contributing cause of DMO.

4. Any ocular pathology or ocular condition other than diabetes that, in the opinion of the investigator, might affect macular oedema.

5. History of any therapy (anti-vascular endothelial growth factor (VEGF) treatment, focal/grid macular photocoagulation, intravitreal or peribulbar corticosteroids) for DMO or diabetic retinopathy in the past 12 months.

6. History of YAG capsulotomy performed within 2 months before enrolment.

7. Aphakia.

8. Evidence of glaucoma or ocular hypertension under topical medication.

\section{Scheduling}

SD-OCT scans (described below) were performed at each visit in both Groups. Age, gender and diabetes duration were recorded at baseline. Glycemia and glycated haemoglobin values were recorded at baseline and at 2 months after surgery. Best-corrected visual acuity (BCVA) was measured by Early Treatment Diabetic Retinopathy Study (ETDRS) charts [12] at baseline in both groups and at each visit for Group 1, but only at month 6 for Group 2 (except in the case of dropout, see below). All visits had to be performed within 1 week pre or post the scheduled time.

\section{Method of acquisition of OCT images}

All the SD-OCT images were acquired using a 9-mm-length high-resolution scan using the 'radial scan' modality, centred to the foveola. The presence of E-DMO was judged from the scan crossing the foveola with the worse morphology or, in the case of media opacities, with the best visibility of the retinal structure. Normative retinal ETDRS maps, available in every SD-OCT machine, were used to provide quantitative measurement of the CST indicated in the $500-\mu \mathrm{m}$-radius ring centred to the foveola, and the evaluation of thickening less than $30 \%$ of maximum normal levels that defined E-DMO. The OCT machines used by each centre, plus their nomograms, are listed in Supplementary Table 1. Each centre used just one OCT brand for all observations.

\section{Clinical significance}

Changes in CST $<10 \mu \mathrm{m}$ and BCVA $<5$ letters were considered to be either an artefact or part of the normal fluctuation. Any change below these values was not considered to be clinically significant and therefore not reported in the results.

\section{Data collection}

A specific database was illustrated and distributed to all centres. Collected data were sent to the coordinating centre on a monthly basis.

\section{Surgery and medications}

The topical, local or systemic use of nonsteroidal anti-inflammatory drugs (NSAIDs) was not permitted during the period before cataract surgery and throughout the study. Preoperative therapy was standard of care for cataract surgery; iodopovidone eye drops and/or topical antibiotics were used at the investigator's discretion. Cataract surgery was conducted by phacoemulsification according to the surgeon's usual routine. The postoperative therapy was identical in all centres: an association of topical steroid and antibiotic drops four times a day for 2 weeks, reduced to twice a day for 1 week and once a day the third week.

\section{Study participant withdrawal}

A clinically significant worsening of E-DMO, defined as an increase in CST of $\geq 50 \mu \mathrm{m}$ on OCT imaging compared to last preoperative values in Group 1 or baseline values in Group 2, with or without BCVA loss, prompted the withdrawal from the study, to allow a therapeutic regimen established at the investigator's discretion. In the case of a patient with both eyes under investigation, the second eye continued to receive the scheduled followup.

\section{Statistical analysis}

Data were reported by means of descriptive statistics. Quantitative variables were summarised by means and standard deviations; qualitative items with absolute counts and percentages. Student's $t$-test was used to assess differences in mean values, unpaired if referred to independent groups, and paired when considering bilateral eyes. Association between 
Table 2. Summary of results in Groups 1 and 2.

\begin{tabular}{|c|c|c|c|}
\hline & Group 1 & Group 2 & Difference ( $p$ values) \\
\hline Eyes & 78 & 65 & \\
\hline Lost to follow-up & 6 & 4 & 0.453 \\
\hline \multicolumn{4}{|l|}{ CST worsening } \\
\hline$\geq 10 \mu \mathrm{m}$ & $80.7 \%$ (63 eyes) & $44.6 \%$ (29 eyes) & $<0.0001$ \\
\hline$\geq 50 \mu \mathrm{m}$ & $65.45 \%$ (51 eyes) & $15.4 \%$ (10 eyes) & $<0.0001$ \\
\hline$\geq 100 \mu \mathrm{m}$ & $26.9 \%$ (21 eyes) & $3.07 \%$ (2 eyes) & $<0.0001$ \\
\hline BCVA loss (mean) & $8.2 \pm 6.2$ & $2.6 \pm 3.5$ & $<0.0001$ \\
\hline
\end{tabular}

A

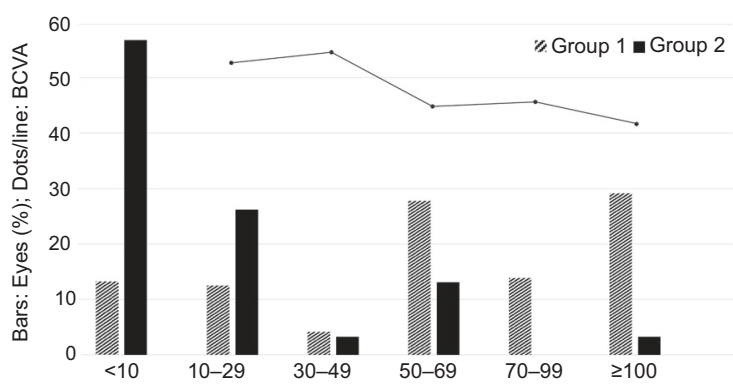

B

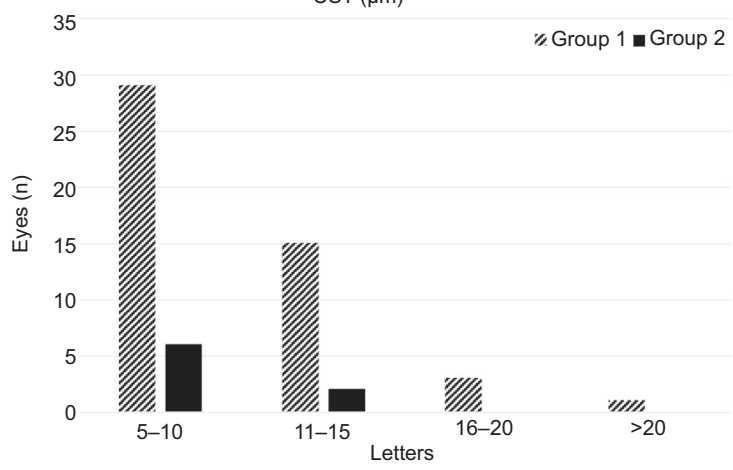

C

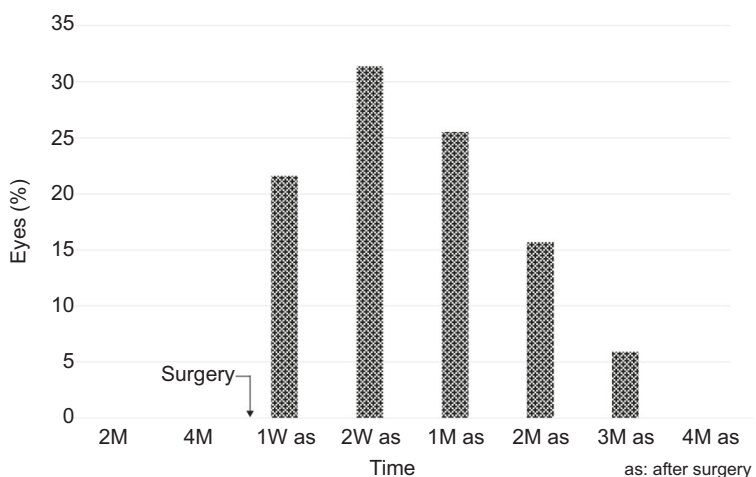

Fig. 2 Amount and timing of worsening of BCVA and CST after cataract surgery in eyes with E-DMO. Compared to natural history (Gr 2) cataract surgery $(\mathrm{Gr} 1)$ determined a marked increase in CST and decrease in BCVA peaked at 15-30 days postop. A Correlation between worsening of postoperative BCVA and CST in Group 1 (line) and distribution of CST worsening among the two groups during follow-up, divided in six progressive classes (bar chart). Most of the eyes in Group 2 (follow-up) evidenced a minimal or mild CST worsening, less than 50 microns in $84 \%$ of eyes, while $65.4 \%$ of eyes of Group 1 (surgery) evidenced a CST worsening higher than 50 microns $(p<0.0001)$. B Comparison of worsening of BCVA in the two groups during follow-up. In Group 1 worsening was calculated relative to the first postoperative measurement at 1 week. C Percentage of eyes in Group 1 with CST worsening $\geq 50 \mu \mathrm{m}$ according to time of postoperative follow-up. The vast majority of eyes $(78.4 \%)$ worsened during the first postoperative month. qualitative items was analysed with the $x^{2}$ test. A linear logistic model with a multivariate approach was used to evaluate independent covariates associated with worsening. Time on study was analysed with the Kaplan-Meier method and differences between the two curves were assessed by the log-rank test.

We intended to enrol 300 eyes, based on the hypothesis of detecting a $\geq 20 \mu \mathrm{m}$ worsening on OCT in $40 \%$ of eyes in Group 1 . However, statistically relevant differences in this measure were detected before 300 eyes were enrolled, so recruitment was halted at that point.

\section{RESULTS}

A total of 78 eyes, from 78 patients were enrolled in Group 1, and 65 eyes of 65 patients in Group 2 (35 eyes were phakic, 30 were pseudophakic). Both eyes were enrolled in 16 patients due to the presence of bilateral E-DMO. In these patients, one was eye scheduled for cataract extraction (Group 1), and the contralateral was used as control (Group 2). In Group 1, six eyes were lost to follow-up, three at the first postoperative month, and three at the second month postoperatively. In Group 2, four eyes were lost to follow-up, two at month 4 , and two at month 8 .

\section{Baseline characteristics}

Participants' demographics, diabetic and ocular comorbidities (Table 1) were similar in both groups, other than mean BCVA, which was lower in Group 1 thanks to the presence of cataract (32.4 \pm 5.7 letters in Group 1 vs $48.0 \pm 5.5$ letters in Group 2, $p<$ 0.0001 ). Mean age was $72.7 \pm 5.1$ in Group 1 and $68.7 \pm 8.1$ in Group 2. Diabetes duration was $12.8 \pm 7.2$ (median:12; range: $1-35$ ) in Group 1 and $16.1 \pm 11.0$ (median:14; range: 2-52) in Group 2. Insulin use was $33.3 \%$ of patients in Group 1 and $40.8 \%$ in Group 2. Glycaemic values were $129.3 \pm 31.3$ and glycate haemoglobin values were $51.9 \pm 11.3$ in Group 1 and $127.8 \pm$ 32.6 and $53.9 \pm 8.5$ in Group 2. Mean CST was $291.6 \pm 44.5$ in Group 1 and $295.1 \pm 29.8$ in Group 2. Other than VA, no differences in any other baseline parameters were statistically significant.

\section{Follow-up}

In both groups, 6-month glycaemic values were not significantly different from those measured at baseline, suggesting that glycaemic fluctuations did not influence the results.

The differences in terms of CST and BCVA worsening between Group 1 and 2 are summarised in Table 2.

\section{Group 1 eyes}

During the preoperative 4 months of follow-up, no significant variations in CST were noted in the 78 eyes in Group 1. Conversely, during the 4 months of follow-up after cataract surgery, CST worsened $\geq 10 \mu \mathrm{m}$ in 63 eyes $(80.7 \%)$ with a corresponding BCVA loss (mean loss $8.4 \pm 6.2$ letters, Fig. 2A). In 51 eyes (65.4\%), CST worsening was $\geq 50 \mu \mathrm{m}$ with a postoperative BCVA decrease of 10 \pm 5.4 letters. In accordance with the protocol, these eyes were promptly withdrawn from the study. In 21 eyes (26.9\%), CST worsened $\geq 100 \mu \mathrm{m}$, with a VA loss of $12.9 \pm 6.7$ letters (Fig. 2A, B). 
CST worsened by $30-49 \mu \mathrm{m}$ in 3 eyes (3.4\%) and a by $10-29 \mu \mathrm{m}$ in 9 eyes (11.5\%), but no significant BCVA changes were noted in either of these subgroups.

The negative impact of surgery on CST was evident from the first postoperative week, peaked during the first month and then rapidly decreased afterward. Among the 51 eyes that worsened $\geq 50 \mu \mathrm{m}, 40$ eyes (78.4\%) were withdrawn during the first month: 11 eyes $(21.6 \%)$ at week 1,16 eyes (31.4\%) at week 2 and 13 eyes $(25.5 \%)$ at month 1 . After the first postoperative month, this number dramatically decreased with just 8 eyes (15.7\%) withdrawn at month 2, 3 eyes (5.9\%) at month 3 and no eyes withdrew at month 4 (Fig. 2C). Compared with the group as a whole, eyes with CST increases of $>50 \mu \mathrm{m}$ had higher glycaemic values (137.3 \pm 31.2 vs $115 \pm 28.8, p=0.0008)$ and glycated haemoglobin $(54 \pm$ 11.4 vs $46.0 \pm 7.2, p=0.004$ ) at baseline. No correlation was found with age, duration of diabetes or insulin intake or the level of diabetic retinopathy, due to the small number of eyes present in each stage.

\section{Group 2}

During the 8 months of follow-up, E-DMO worsening was observed even in the non-operated group, but in fewer eyes and with a far lower impact on CST and visual function (Fig. 2A, B). A CST worsening of $\geq 10 \mu \mathrm{m}$ was observed in $29 / 65$ eyes $(44.6 \%)$ (compared with $63 / 78$ eyes (80.7\%) of Group $1(p<0.0001)$ ), and just 10 eyes (15.4\%) were withdrawn because of CST worsening $\geq 50 \mu \mathrm{m}$ (compared with 51 eyes $(65.4 \%)$ in Group $1(p<0.0001)$ ). Moreover, mean CST worsening of Group 2 was lower compared to Group $1(38.6 \pm 30.4 \mu \mathrm{m}$ vs $85.5 \pm 55.3 \mu \mathrm{m}, p<0.0001)$ with a lower BCVA loss $(-2.6 \pm 3.5$ letters vs $-8.2 \pm 6.2$ letters, $p<0.0001)$. The greatest CST worsening observed was $252 \mu \mathrm{m}$ in Group 1, and $125 \mu \mathrm{m}$ in Group 2, and the greatest BCVA loss was 39 in Group 1 and 11 letters in Group 2. No relationship between baseline and diabetic parameters and CST worsening of $\geq 50 \mu \mathrm{m}$ was evident in Group 2.

\section{Bilateral patients}

The negative influence of cataract surgery was particularly evident in the 16 patients followed for bilateral E-DMO, in which one eye was operated on at the fourth month, and the other eye followed without intervention. Because some Group 2 eyes already had cataract with decreased VA were listed for surgery after the end of the study, the only significant parameter to evaluate the impact of surgery in this subgroup is worsening of CST. In 14 patients $(87.5 \%)$ the operated eye worsened more than the non-operated eye of $\geq 10 \mu \mathrm{m}$. Among the 16 operated eyes, 13 (81.2\%) worsened $\geq 50 \mu \mathrm{m}$ and were withdrawn from the study compared with 4 eyes $(25 \%)$ in the control group $(p=0.001)$. Three patients worsened in both eyes; one patient worsened just in the nonoperated eye.

\section{DISCUSSION}

Cataract develops faster and earlier in people with diabetes, compared with those with normal glycemia [4]. As a consequence, cataract surgery is a very frequently performed procedure in people with diabetes. A recent epidemiologic study, DICAT I, found that $27 \%$ of patients undergoing cataract surgery were diabetic [7].

The presence of diabetes is a negative prognostic factor for cataract surgery independently of preoperative macular impairment. Even in diabetic eyes without DMO, the risk ratio of developing oedema compared to non-diabetics rises from 1.8 in subjects without any sign of retinopathy to 10.3 in the presence of advanced stages [10]. Topical NSAID use during the first months postoperatively reduces this risk [13]. According to DICAT I, $25 \%$ of diabetic subjects undergoing cataract surgery have preoperative DMO [7]; this confers a high risk of macular worsening, potentially eliminating the vision benefits of cataract extraction $[8,10]$. Intravitreal steroid or anti-VEGF therapy immediately before or even at the end of cataract surgery is very effective in reducing this complication [14, 15]. However, in clinical practice, the presence of DMO is considered to be 'clinically relevant' and the object of therapeutic attention only when a consistent increase in macular thickness, associated with alterations in retinal structure, is evident at OCT examination. The presence of one or a few microcysts often positioned at the foveal side and associated with minimal thickening (so-called non-clinically significant oedema, or pre-clinical oedema) is not normally of interest.

The ESASO OCT DMO classification [11] describes four progressive stages of $\mathrm{DMO}$, according to the presence and grading of seven OCT biomarkers, with E-DMO representing the first manifestation of the disease (Fig. 1). This study demonstrates that E-DMO slowly worsens in less than 1 year, and that this worsening may become clinically relevant in over $10 \%$ of cases. Cataract surgery dramatically worsens this course in the vast majority of cases: in this study, 4 months after surgery, 51 eyes (65.4\%) experienced CST worsening $\geq 50 \mu \mathrm{m}$ with a visual loss $>10$ letters, which necessitated these patients being immediately withdrawn from the study, and started on appropriate therapy, per investigator's choice (Table 2). Even forms of E-DMO with just one to two microcysts (such as those depicted in Fig. 1A) may develop a clinically significant worsening (Fig. 3). The highly negative effect of E-DMO was particularly obvious in the limited number of 16 bilateral patients, where systemic conditions could not confound the comparison: in 14 patients (87.5\%) the operated eye worsened more than the non-operated eye of at least $10 \mu \mathrm{m}$, and in 13 patients $(81.2 \%)$ the operated eye was withdrawn for CST worsening $>50 \mu \mathrm{m}$. These results demonstrate that the negative impact of cataract surgery on E-DMO occurs very rapidly, lasts about 1 month, with the effects declining rapidly after the second month (Fig. 2C). Higher baseline glycaemic values were a risk factor for oedema worsening after surgery.

As noted earlier, topical NSAID use was avoided throughout the study, even though these agents may reduce the risk of developing postoperative macular oedema in both diabetic and non-diabetic eyes with normal preoperative maculae. No benefit
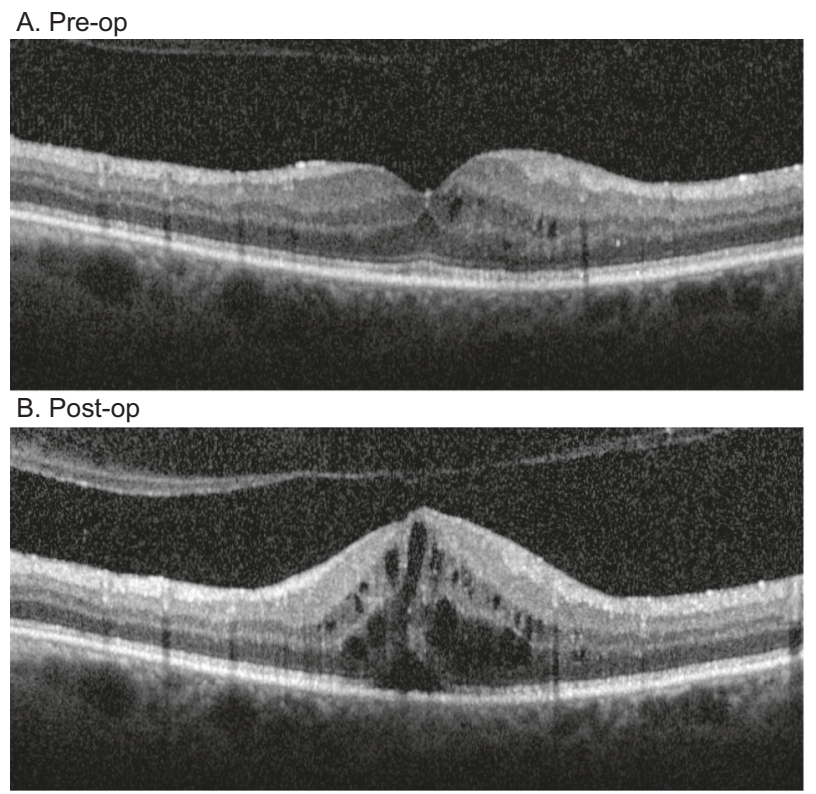

Fig. 3 Worsening of minimal E-DMO after cataract surgery. A Preoperative oedema, CST $378 \mu \mathrm{m}$, BCVA 52 letters. B Two weeks after surgery: CST $630 \mu \mathrm{m}$ and BCVA 39 letters $(-13)$. 
of NSAID use has previously been demonstrated in the presence of preoperative oedema, but their use may have had some effect even in eyes with preoperative E-DMO, and this would generate a non-measurable bias in the incidence and/or clinical relevance of E-DMO worsening. To avoid this potential bias, we decided to avoid their use.

It is impossible to compare our results with those of previous similar studies, because as a recent editorial pointed out 'real-world data on the outcomes of cataract surgery with co-existent DME is currently lacking' [16]. A number of descriptions of worsening DMO after cataract surgery exist, but this is largely old literature $[8,9]$, and few trials used OCT for precise CST measurement. Given the recent coining of the term, no trials have previously examined eyes with E-DMO. In one prospective multicentre trial, the DRCR network studied 266 diabetic eyes with non-centre-involving DMO undergoing cataract surgery, and observed that centreinvolving DMO developed in $11.5 \%$ of eyes [17]. In another smaller prospective monocentre trial involving patients with diabetes who underwent cataract surgery, Chen et al. found worsening of DMO in $13 / 20(65 \%)$ eyes enrolled [18]. Another feasibility trial conducted by the DRCR network that involved 63 eyes failed to generate conclusive results due to a lack of standardisation of DMO management among the involved centres [19].

This study has a number of limitations. The COVID-19 pandemic restricted study enrolment; a more complete enrolment may have revealed greater insight. The follow-up period, particularly in patients in Group 2, although long enough for evaluating the study hypothesis, was not sufficiently long enough to properly evaluate the natural course of the disease in these patients. For this reason, we plan to further follow these eyes for a total period of 18 months.

This study not only reinforces the importance of close pre- and postoperative monitoring of the maculae of patients with diabetes undergoing cataract surgery, but also focuses attention for the first time on what was previously considered an insignificant form of the disease, E-DMO, and highlights how even than this minimal oedema may be dramatically worsened by cataract surgery.

Supplementary information is available on Eye's website.

\section{Summary}

What was known before

- Around one in five people who undergo cataract surgery have diabetes.

- If a person has DMO, cataract surgery can increase intravitreal inflammatory mediators and exacerbate the oedema, worsening vision.

- It is unclear whether cataract surgery is associated with this effect in eyes with 'subclinical' or 'early' DMO.

What this study adds

- This study shows that the presence of early DMO prior to cataract surgery is associated with worsening of macular oedema and vision loss.

- Close monitoring of the retina of diabetic patients before and after cataract surgery is warranted, even in patients with minimal oedema.

\section{REFERENCES}

1. International Diabetes Federation. IDF Diabetes Atlas Ninth Edition. https://www. diabetesatlas.org/upload/resources/material/20200302_133351_IDFATLAS9efinal-web.pdf (2019). Accessed 20 June 2021.
2. Selvin E, Coresh J, Brancati FL. The burden and treatment of diabetes in elderly individuals in the U.S. Diabetes Care. 2006;29:2415-9.

3. Wang Q, Zhang X, Fang L, Guan Q, Guan L, Li Q. Prevalence, awareness, treatment and control of diabetes mellitus among middle-aged and elderly people in a rural Chinese population: a cross-sectional study. PLoS One. 2018;13: e0198343.

4. Pollreisz A, Schmidt-Erfurth U. Diabetic cataract-pathogenesis, epidemiology and treatment. J Ophthalmol. 2010;2010:608751.

5. International Diabetes Federation. Clinical practice recommendations for managing diabetic macular edema. https://www.idf.org/component/attachments/?task $=$ download\&id=2153 (2019). Accessed 20 June 2021.

6. Shin ES, Sorenson CM, Sheibani N. Diabetes and retinal vascular dysfunction. J Ophthalmic Vis Res. 2014;9:362-73.

7. Panozzo G, Staurenghi G, Dalla Mura G, Giannarelli D, Alessio G, Alongi S, et al. Prevalence of diabetes and diabetic macular edema in patients undergoing senile cataract surgery in Italy: The Dlabetes and CATaract study. Eur J Ophthalmol. 2020;30:315-20.

8. Dowler JG, Hykin PG, Lightman SL, Hamilton AM. Visual acuity following extracapsular cataract extraction in diabetes: a meta-analysis. Eye (Lond). 1995;9:313-7. Pt 3

9. Gupta A, Gupta V. Diabetic maculopathy and cataract surgery. Ophthalmol Clin North Am. 2001;14:625-37.

10. Chu CJ, Johnston RL, Buscombe C, Sallam AB, Mohamed Q, Yang YC. Risk factors and incidence of macular edema after cataract surgery: a database study of 81984 eyes. Ophthalmology. 2016;123:316-23.

11. Panozzo G, Cicinelli MV, Augustin AJ, Battaglia Parodi M, Cunha-Vaz J, Guarnaccia $\mathrm{G}$, et al. An optical coherence tomography-based grading of diabetic maculopathy proposed by an international expert panel: The European School for Advanced Studies in Ophthalmology classification. Eur J Ophthalmol. 2020;30:8-18.

12. ETDRS Group. Grading diabetic retinopathy from stereoscopic color fundus photographs-an extension of the modified airlie house classification: ETDRS Report Number 10. Ophthalmol. 1991;98:786-806.

13. Yüksel B, Karti Ö, Kusbeci T. Topical nepafenac for prevention of post-cataract surgery macular edema in diabetic patients: patient selection and perspectives. Clin Ophthalmol. 2017;11:2183-90.

14. Furino C, Boscia F, Niro A, D'Addario M, Grassi MO, Saglimbene V, et al. Diabetic macular edema and cataract surgery: phacoemulsification combined with dexamethasone intravitreal implant compared with standard phacoemulsification. Retina. 2021;41:1102-9.

15. Rauen PI, Ribeiro JA, Almeida FP, Scott IU, Messias A, Jorge R. Intravitreal injection of ranibizumab during cataract surgery in patients with diabetic macular edema. Retina. 2012;32:1799-803.

16. Goh JKS, Lim LL. Dealing with co-existent cataract and diabetic macular oedema: an increasingly common conundrum now solved? Clin Exp Ophthalmol. 2020:48:424-6.

17. Baker CW, Almukhtar T, Bressler NM, Glassman AR, Grover S, Kim SJ, et al. Macular edema after cataract surgery in eyes without preoperative central-involved diabetic macular edema. JAMA Ophthalmol. 2013;131:870-9.

18. Chen XY, Song WJ, Cai HY, Zhao L. Macular edema after cataract surgery in diabetic eyes evaluated by optical coherence tomography. Int J Ophthalmol. 2016;9:81-5.

19. Bressler SB, Baker CW, Almukhtar T, Bressler NM, Edwards PA, Glassman AR, et al. Pilot study of individuals with diabetic macular edema undergoing cataract surgery. JAMA Ophthalmol. 2014;132:224-6.

\section{ACKNOWLEDGEMENTS}

The authors wish to thank Mark Hillen, PhD, for assistance in the preparation of this manuscript.

\section{AUTHOR CONTRIBUTIONS}

GP and GDM conceived and designed the presented study. VA, AA, SC, PC, CC, RDA, $F F, M N, M R P$ and $C P$ performed the data collection. GP, GDM, DG, EF and EG performed the analysis. GP and GDM wrote the manuscript. FB, GA, GM, LM, SS and DT provided critical review of the manuscript.

\section{COMPETING INTERESTS}

The authors declare no competing interests. 


\section{ADDITIONAL INFORMATION}

Supplementary information The online version contains supplementary material available at https://doi.org/10.1038/s41433-021-01718-4.

Correspondence and requests for materials should be addressed to G.P.
Reprints and permission information is available at http://www.nature.com/ reprints

Publisher's note Springer Nature remains neutral with regard to jurisdictional claims in published maps and institutional affiliations. 